\title{
Renal tubular epithelial cells of the native hibernator Syrian hamster recover more rapidly from endoplasmic reticulum stress compared to those of human or mouse following warm anoxia-reoxygenation, possibly due to increased proteasomal function
}

\author{
THEODOROS ELEFTHERIADIS, GEORGIOS PISSAS, GEORGIAANTONIADI, GEORGIOS FILIPPIDIS, \\ VASSILIOS LIAKOPOULOS and IOANNIS STEFANIDIS
}

Department of Nephrology, Faculty of Medicine, University of Thessaly, 41110 Larissa, Greece

Received October 29, 2018; November 28, 2018

DOI: $10.3892 /$ wasj.2018.4

\begin{abstract}
Comparative biology may reveal novel therapeutic strategies against human diseases. Ischemia-reperfusion (IR) injury induces a number of diseases. It is known that hibernating mammals survive IR since during hibernation, prolonged periods of torpor with a marked decrease in blood flow and breathing rate are interrupted by short periods of arousal. In the present study, the differences in the characteristics of endoplasmic reticulum (ER) stress and the subsequent unfolded protein response, which are induced by IR and may cause cell death among humans, mice or the native hibernator Syrian hamster were examined in vitro using renal proximal tubular epithelial cells (RPTECs) derived from these three sources. RPTECs were subjected to anoxia or reoxygenation, both at $37^{\circ} \mathrm{C}$. Cell death was measured by $\mathrm{LDH}$ release assay. ER stress was assessed by determining the levels of phosphorylated protein kinase RNA-like ER kinase, ubiquitinated proteins and $\mathrm{Bcl}-2$-associated $\mathrm{X}$ protein ( $\mathrm{Bax}$ ) by western blot analysis. For proteasomal activity, a specific assay was used. The results revealed that anoxia induced ER stress in all the evaluated RPTECs, from which only the hamster-derived RPTECs recovered during reoxygenation. Anoxia and reoxygenation increased protein ubiquitination in the human- and mouse-derived RPTECs, whereas this was decreased in the hamster-derived RPTECs. Anoxia enhanced proteasomal activity in all the evaluated RPTECs. In the human- and mouse-derived RPTECs, reoxygenation reduced proteasomal activity, which remained high in the hamster-derived RPTECs.
\end{abstract}

Correspondence to: Dr Theodoros Eleftheriadis, Department of Nephrology, Faculty of Medicine, University of Thessaly, Neo Ktirio, Mezourlo Hill, 41110 Larissa, Greece

E-mail: teleftheriadis@yahoo.com

Key words: hibernation, ischemia-reperfusion, endoplasmic reticulum stress, unfolded protein response, cell death, acute kidney injury
Anoxia and reoxygenation increased Bax expression and induced cell death in the human- and mouse-derived RPTECs, while neither Bax overexpression nor cell death occurred in the hamster-derived RPTECs. Thus, on the whole, the findings of this study demonstrate that compared to human- or mouse-derived RPTECs, those derived from the hamster recover more rapidly from ER stress following warm anoxia-reoxygenation, possibly due to increased proteasomal function.

\section{Introduction}

Ischemia, induced by a reduced blood supply due to either an obstructed artery or a low effective blood volume, plays a significant role in the pathogenesis of a number of human diseases. Even after the restoration of blood flow, the re-entry of oxygen into the ischemic area may aggravate tissue damage by promoting the massive production of reactive oxygen species. This sequence of events occurs in a number of pathological entities, such as coronary heart disease, cerebral ischemia and multiple organ failure (1-3). The kidney is very sensitive to ischemia-reperfusion (IR) injury as in this organ, the partial oxygen pressure is already low under physiological conditions and the energy demands of tubular epithelial cells are also high $(4,5)$.

Certain mammals fall in hibernation in order to cope with the food shortage during winter. Hibernation is characterized by prolonged periods of torpor, during which animals decrease their metabolic rate and temperature, and exhibit profound bradycardia, reduced blood pressure and a breathing rate that cannot be distinguished from apnea by an inexperience observator. Torpor periods are interrupted by short periods of arousal during which the animals become euthermic and restore blood pressure, heart and breathing rates. However, animals survive these cycles of IR without any profound injury, acute kidney injury (AKI) included $(6,7)$. The fact that hibernators are also less sensitive to warm IR injury than phylogenetically related non-hibernators $(8,9)$, as well as the discovery of certain primates that fall in hibernation in a relatively high ambient 
temperature (10), make the study of hibernation interesting from a clinical point of view. Understanding the mechanisms that provide a way of escaping warm IR injury in hibernating mammals, may reveal novel strategies for preventing IR injury in humans.

One of the events that take place under IR is endoplasmic reticulum (ER) stress that is the accumulation of misfolded proteins. ER stress triggers the unfolded protein response (UPR), an adaptive mechanism that aims to restore protein homeostasis. Under normal conditions, the three transmembrane ER mediators of the UPR, protein kinase RNA-like ER kinase (PERK), activating transcription factor 6 (ATF6) and inositol-requiring enzyme 1 (IRE1) are maintained in an inactive state through their association with the ER chaperone binding immunoglobulin protein (BiP). In the case of ER stress, BiP binds the accumulated misfolded proteins and dissociates from PERK, ATF6 and IRE1, leading to their activation. The consequent UPR consists of the attenuation of protein translation through the phosphorylation of eukaryotic initiation factor $2 \alpha$ (eIF $2 \alpha$ ) by the phosphorylated and activated PERK, an increased expression of ER chaperones, and ER-associated protein degradation to remove the misfolded proteins from the ER (11). During ER-associated protein degradation, ATF6 and IRE1 upregulate the ER transmembrane homolog to yeast Hrd1p, which acts as a ubiquitin ligase (E3). This ligase polyubiquitinates the misfolded proteins that translocate to the cytosol for degradation by the proteasome (12). Although the UPR is an adaptive mechanism aiming to degrade misfolded proteins and restore ER homeostasis, if the initial stimulus is severe or prolonged, it can then induce cell death $(11,13)$. In this cell death pathway, PERK, IRE1 and ATF6 upregulate the transcription factor $\mathrm{C} / \mathrm{EBP}$ homologous protein (CHOP), which in turn upregulates various pro-apoptotic proteins, the $\mathrm{Bcl}-2$ associated $\mathrm{X}$ protein (Bax) included $(14,15)$. Accumulating evidence implicates ER-induced cell death in the pathogenesis of a number of disorders $(11,13)$, chronic kidney disease (16) and ischemic AKI included (17).

Since IR triggers ER stress-induced cell death in myocardial $(18,19)$, neural $(20)$, and interestingly, in renal tubular epithelial cells $(21,22)$, this study aimed to evaluate the unique characteristics of IR-induced ER stress, and specifically of ER-associated protein degradation, in renal proximal tubular epithelial cells (RPTECs) of the native hibernator Syrian hamster compared to human-derived RPTECs. Mouse-derived RPTECs were also evaluated as a phylogenetic control for hamster-derived RPTECs.

\section{Materials and methods}

Cell culture conditions and imaging. Primary human RPTECs (cat. no. 4100, ScienCell, Carlsbad, CA, USA), primary Syrian hamster RPTECs (cat. no. HM-6015) and primary C57BL/6 mouse RPTECs (cat. no. C57-6015) were cultured in Complete Epithelial Cell Medium/w kit (cat. no. M6621) (all from Cell Biologics, Chicago, IL, USA), supplemented with epithelial cell growth supplement, antibiotics and fetal bovine serum.

The cells were cultured in 96-well plates at a number of 10,000 cells per well, in 12 -well plates at a number of 100,000 cells per well or in 6-well plates at a number of 300,000 cells per well. The GasPak ${ }^{\mathrm{TM}}$ EZ Anaerobe Container
System with Indicator (cat. no. 26001; BD Biosciences, S. Plainfield, NJ, USA) was used to reduce oxygen levels to $<1 \%$. Cells within the anaerobe container were cultured at $37^{\circ} \mathrm{C}$. These anoxic conditions imitate warm ischemia. Reoxygenation experiments began after a certain period of anoxia. In these experiments, the cells were washed, and fresh supplemented culture medium was added, and the cells were then placed at $37^{\circ} \mathrm{C}$ in a humidified atmosphere containing $5 \% \mathrm{CO}_{2}$. These reoxygenation conditions replicate warm reperfusion. In the case that cell manipulation under anoxic conditions was necessary, this was performed in an anoxic chamber at $37^{\circ} \mathrm{C}$ in a humidified atmosphere containing $<0.1 \% \mathrm{O}_{2}$ and $5 \% \mathrm{CO}_{2}$ (InvivoO 200 hypoxia workstation; Baker Ruskinn, Sanford, ME, USA).

Initially, the difference in the sensitivity of human-, mouseor hamster-derived RPTECs to warm anoxia or reoxygenation was assessed by cell imaging in cells cultured in 12-well plates. For this purpose, an inverted microscope (Axiovert $40 \mathrm{C}$; Carl Zeiss Light Microscopy, Göttingen, Germany) and a digital camera with the related software (3MP USB2.0 Microscope Digital Camera; AmScope, Irvine, CA, USA) were used.

As live cells were required, the various other parameters of the study were evaluated at half of the time needed for detecting severe cell deterioration with cell imaging under anoxia or reoxygenation. Particularly for the hamster cells, which proved remarkably resistant to cell death, the same time points as those for mouse cells were used. All the experiments were performed 9 times.

Biochemical evaluation of cell death. The cells were cultured in 96-well plates as described above. Cell death was assessed colorimetrically by LDH release assay using the Cytotox Non-Radioactive Cytotoxic Assay kit (cat. no. G1780; Promega Corp., Madison, WI, USA). All experiments were performed 9 times.

Evaluation of proteins of interest. The cells were cultured in 6-well plates as described above. The cells were lysed using the T-PER tissue protein extraction reagent (Thermo Fisher Scientific Inc., Rockford, IL, USA) supplemented with protease and phosphatase inhibitors (Sigma-Aldrich; Merck Millipore, Darmstadt, Germany and Roche Diagnostics, Indianapolis, IN, USA, respectively). Protein was quantified via Bradford assay (Sigma-Aldrich; Merck Millipore) and $10 \mu \mathrm{g}$ from each sample were used for western blot analysis. Protein samples were electrophoresed in a 4-12\% Bis-Tris acrylamide gel (NuPAGE 4-12\% Bis-Tris Gel 1.0 mm x 15 well; cat. no. NP0323BOX; Invitrogen/Thermo Fisher Scientific, Inc.) at $180 \mathrm{~V}$ constant for $30 \mathrm{~min}$. Blotting of the electrophoresed gel proteins on the polyvinylidene difluoride (PVDF) membrane was performed via electroporation at $30 \mathrm{~V}$ constant for $1 \mathrm{~h}$. Skimmed milk in Tris-buffered saline with Tween-20 was used for blocking. The blots were incubated with the primary antibody against the phosphorylated at threonine 981 PERK (p-PERK) (cat. no. E-AB-21296; Elabscience Biotechnology Inc., Houston, TX, USA), ubiquitin (cat. no. 3933; Cell Signaling Technology, Danvers, MA, USA), Bax (cat. no. ab32503; Abcam, Cambridge, UK), and $\beta$-actin (cat. no. 4967; Cell Signaling Technology) for $16 \mathrm{~h}$ at $4{ }^{\circ} \mathrm{C}$, followed by a 30 -min incubation at room temperature with the secondary antibody (anti-rabbit 
IgG, HRP-linked antibody, cat. no. 7074; Cell Signaling Technology). In the case of the re-probing of the PVDF blots, the previous primary and secondary antibody were removed with the Restore Western Blot Stripping Buffer (Thermo Fisher Scientific, Inc.) according to the manufacturer's instructions. Bands were visualized via enhanced chemiluminescent detection using a LumiSensor Plus Chemiluminescent HRP Substrate kit (GenScript Corporation, Piscataway, NJ, USA). The analysis of the western blots was carried out using ImageJ software (National Institute of Health, Bethesda, MD, USA). All experiments were performed 9 times.

Evaluation of proteasomal activity. Proteasomal activity was assessed by measuring the proteasomal chymotrypsin-like degradation of a specific substrate in the RPTECs cultured in 96-well plates with a luminescent assay. For this purpose, the Proteasome-Glo ${ }^{\mathrm{TM}}$ Chymotrypsin-Like Cell-Based assay (cat. no. G8660/1/2; Promega Corp.) was used according to the manufacturer's instructions. All experiments were performed 9 times.

Statistical analysis. The one-sample Kolmogorov-Smirnov test was used for assessing the normality of the evaluated variables and the one-way analysis of variance (ANOVA) followed by the Bonferroni's correction test for comparison of means. Results are expressed as the means \pm SD and a $\mathrm{P}<0.05$ was considered to indicate a statistically significant difference. Statistical analysis of cell imaging results was performed with the Kruskal-Wallis $\mathrm{H}$ test since these results did not fit to the normal distribution. For the reader's convenience, the results were presented following normalization of means for the respective control groups. IBM SPSS Statistical software for Windows, Version 20 (IBM Corp., Armonk, NY, USA) was used for statistical analysis.

\section{Results}

Cell death due to anoxia or reoxygenation. Cell imaging experiments revealed that the human-derived RPTECs significantly deteriorated at $4 \mathrm{~h}$ of anoxia, the mouse RPTECs were less sensitive to anoxia since they declined at $48 \mathrm{~h}$, while the hamster-derived RPTECs were extremely resistant since they retained their morphology even after 5 days of observation $(\mathrm{P}<0.001$; Fig. $1 \mathrm{~A}$ and $\mathrm{B})$. The human-derived RPTECs significantly deteriorated, exhibiting condensation and a loss of adherence in a very large scale, following $8 \mathrm{~h}$ of reoxygenation. The mouse-derived RPTECs significantly deteriorated at an even earlier time point, at $4 \mathrm{~h}$, whereas the hamster-derived RPTECs resisted reoxygenation since they remained in good condition even after $48 \mathrm{~h}$ of observation $(\mathrm{P}<0.001$; Fig. $1 \mathrm{C}$ and $\mathrm{D})$.

As live cells were required for all the subsequent experiments, and according to the above cell imaging results, the time periods of anoxia or reoxygenation were set at the half of the time needed for the death of human- or mouse-derived RPTCs under conditions of anoxia or reoxygenation. Hence, in all the subsequent experiments, anoxia was applied for $2 \mathrm{~h}$ to the human-derived RPTECs, and for $24 \mathrm{~h}$ to the mouse-derived RPTECs and their phylogenetically related hamster RPTECs. Accordingly, reoxygenation was applied for
$4 \mathrm{~h}$ to the human-derived RPTECs, and for $2 \mathrm{~h}$ to the mouseand hamster-derived RPTECs.

The resistance of the hamster-derived RPTECs to warm anoxia and reoxygenation was also confirmed biochemically by $\mathrm{LDH}$ release assay. Both the human- and mouse-derived RPTECs proved to be sensitive to anoxia or reoxygenation. For the human-derived RPTECs, cell death increased by $271 \pm 16 \%$ under anoxic conditions, and even further by $354 \pm 20 \%$ under conditions of reoxygenation. In the mouse-derived RPTECs, anoxia increased cell death by $197 \pm 6 \%$ and reoxygenation even more so by $282 \pm 16 \%$. On the contrary, the hamster-derived RPTECs resisted cell death due to either anoxia or reoxygenation. Compared to the control hamster-derived RPTECs, cell death was $113 \pm 18 \%$ under conditions of anoxia and $107 \pm 14 \%$ under conditions of reoxygenation (Fig. 1E).

ER stress due to anoxia or reoxygenation. Anoxia increased ER stress, as assessed by the level of p-PERK in all the evaluated RPTECs. Compared to the respective control RPTECs, in the human-derived RPTECs, anoxia increased the p-PERK level by a factor of $1.72 \pm 0.35$, in the mouse-derived RPTECs by a factor of $3.47 \pm 0.28$, and in the hamster-derived RPTECs by a factor of $1.96 \pm 0.74$. Under conditions of reoxygenation, in the human-derived RPTECs, the p-PERK level remained high, $2.13 \pm 1.05$-fold the level of the control. In the mouse-derived RPTECs, p-PERK expression increased further by a factor of $5.47 \pm 2.28$, while in the hamster-derived RPTECs, p-PERK expression decreased at the $0.79 \pm 0.14$ of the level found in the control cells. Thus, under conditions of reoxygenation, and contrary to the human- or mouse-derived cells, the hamster-derived RPTECs recovered more rapidly from ER stress (Fig. 2).

Bax protein expression under conditions of anoxia or reoxygenation. Compared to the respective control RPTECs, anoxia increased Bax expression by a factor of $2.34 \pm 0.20$ in the human-derived RPTECs, and by a factor of $1.43 \pm 0.30$ in the mouse-derived RPTECs. Under conditions of reoxygenation, in the human-derived RPTECs, Bax expression remained high, 2.75 \pm 0.20 -fold the expression seen in the control. In the mouse-derived RPTECs, reoxygenation increased Bax expression further by a factor of $3.47 \pm 1.44$. Neither anoxia nor reoxygenation affected the expression of Bax in the hamster-derived RPTECs, since under anoxic conditions it was $0.92 \pm 0.02$-fold and under conditions of reoxygenation it was $1.02 \pm 0.10$-fold the level found in the control cells (Fig. 3).

Protein ubiquitination and proteasomal activity under conditions of anoxia and reoxygenation. Anoxia and reoxygenation increased the levels of ubiquitinated proteins in the humanand mouse-derived RPRECs. Compared to the control cells, anoxia increased the levels of ubiquitinated proteins by a factor of $1.92 \pm 0.20$ and $1.96 \pm 0.02$ in the human- and mouse-derived RPTECs, respectively. Under conditions of reoxygenation, the levels of ubiquitinated proteins remained high, $2.00 \pm 0.25-$ and $1.51 \pm 0.09$-fold the control in human- and mouse-derived RPTECs, respectively. On the contrary, and compared to the control cells, in the hamster-derived RPTECs, anoxia decreased the levels of ubiquitinated proteins by a factor of $0.79 \pm 0.08$. Reoxygenation decreased levels of ubiquitinated 
A

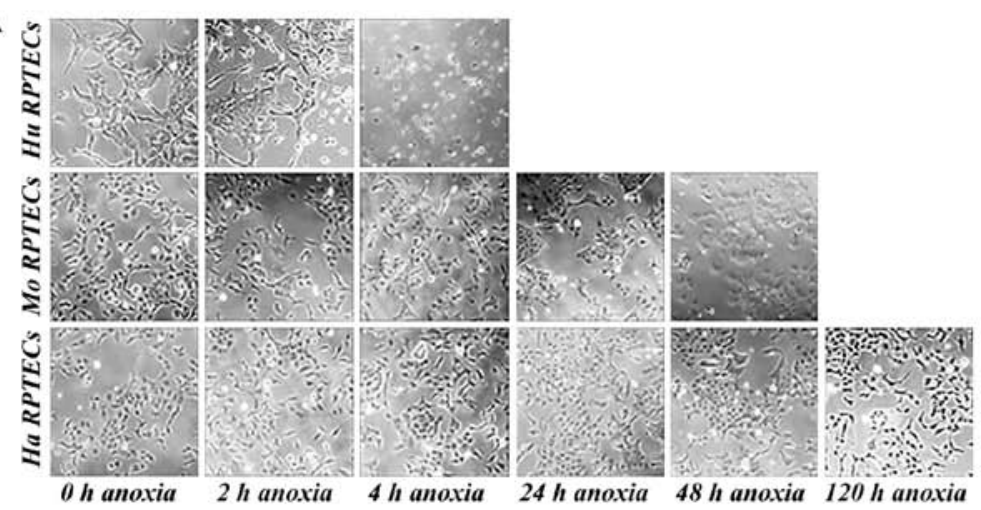

C

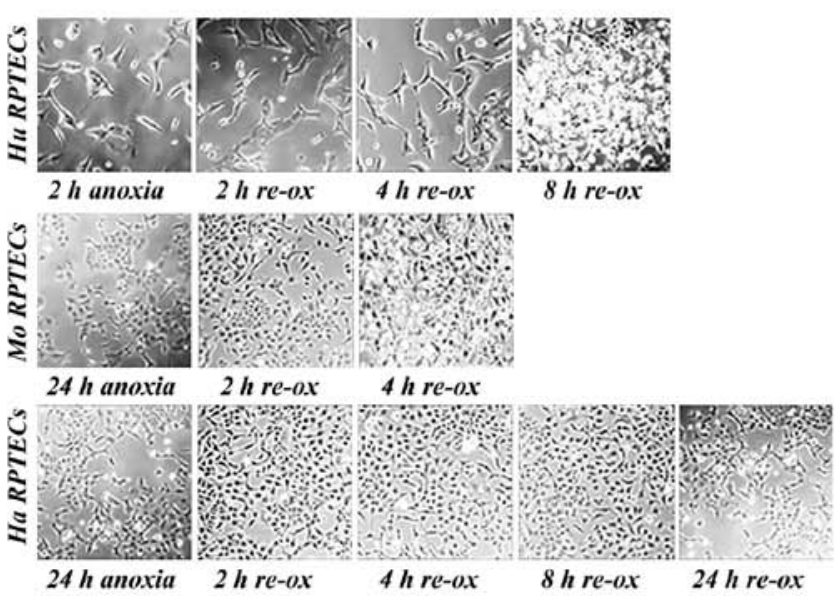

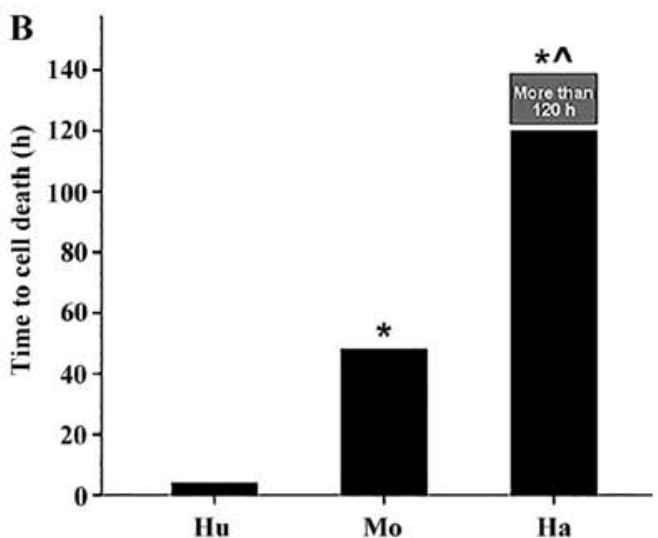

D

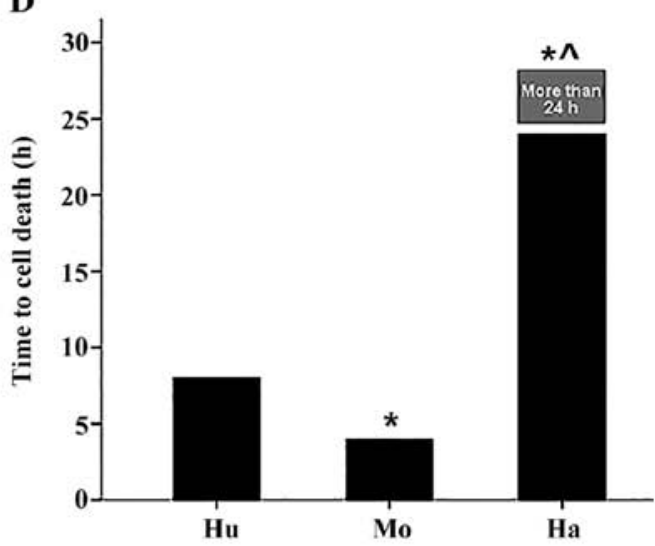

E

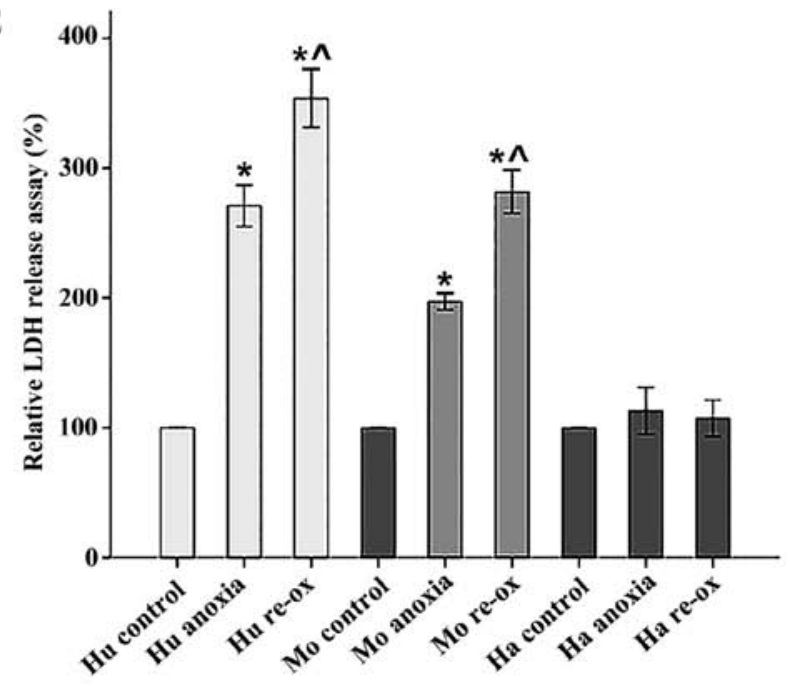

Figure 1. Cell death due to anoxia or reoxygenation. Human, mouse or Syrian hamster-derived RPTECs were cultured under normoxic conditions or subjected to anoxia or anoxia followed by reoxygenation. (A and C) One of the 9 cell immaging experiments for each cell type under anoxic conditions or reoxygenation conditions is depicted. These experiments revealed that compared to the human- or mouse-derived RPTECs, the hamster-derived RPTECs were incredibly resistant to cell death due to (B) anoxia or (D) reoxygenation. ${ }^{*} \mathrm{P}<0.05$, mouse- or hamster-derived RPTECs compared to human-derived RPTECs; ${ }^{\wedge} \mathrm{P}<0.05$, significant difference between hamster- and mouse-derived RPTECs. Error bars are not depicted as the results (hours to death) were the same in all the 9 experiments. (E) LDH release assay also confirmed that both the human- and mouse-derived RPTECs were sensitive to either anoxia or reoxygenation, whereas the hamster-derived RPTECs resisted cell death under both conditions. ${ }^{*} \mathrm{P}<0.05$, compared to the controls cultured under normoxic conditions; ${ }^{\wedge} \mathrm{P}<0.05$, significance between cells under reoxygenation and cells under anoxic conditions. Error bars indicate the means \pm SD. Hu, human; Mo, mouse; Ha, hamster; RPTECs, renal proximal tubular epithelial cells.

proteins even more at $0.57 \pm 0.07$ of the level found in the cells cultured under normoxic conditions (Fig. 4).

Anoxia increased proteasomal activity, assessed by the chymotrypsin-like proteasomal degradation of a specific substrate, by a factor of $3.88 \pm 0.21,1.56 \pm 0.19$ and $2.99 \pm 0.37$ in the human-, mouse- and hamster-derived RPTECs, respectively. In the human-derived RPTECs, reoxygenation that followed anoxia reduced proteasomal activity back to the level found in the control cells and more precisely to $0.94 \pm 0.08$ of the control. In the mouse-derived RPTECs, reoxygenation 
A

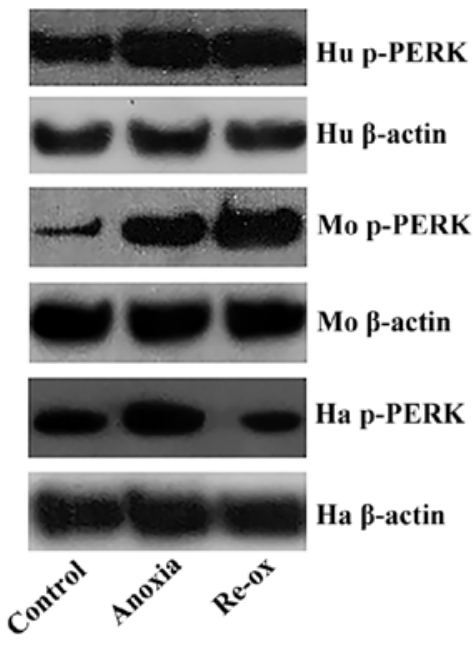

B

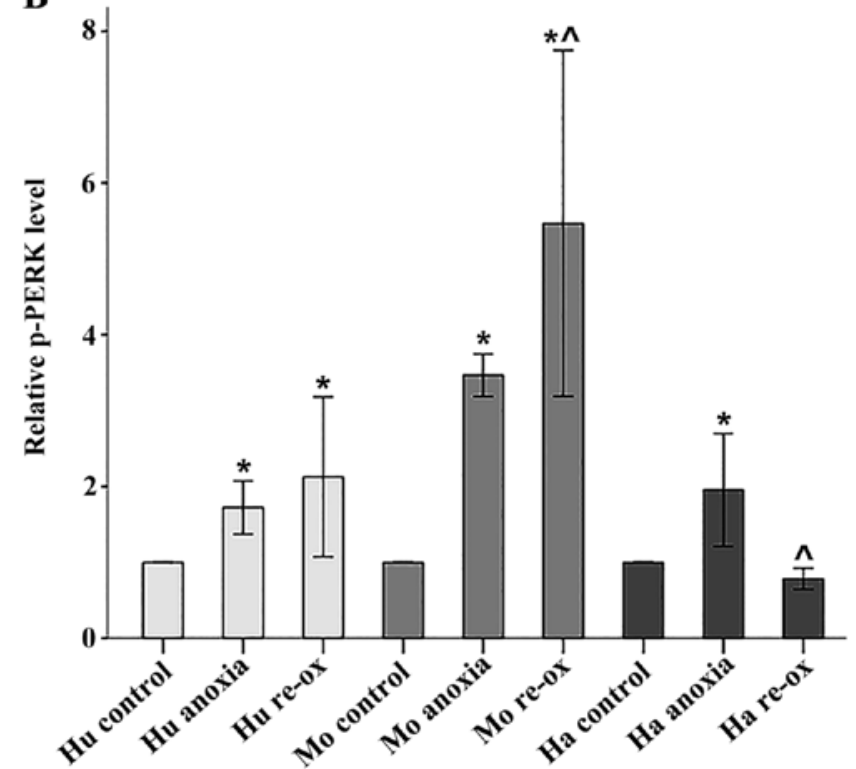

Figure 2. Endoplasmic reticulum stress due to due to anoxia or reoxygenation. Human-, mouse- or Syrian hamster-derived RPTECs were cultured under normoxic conditions or subjected to anoxia or anoxia followed by reoxygenation. (A) Endoplasmic reticulum stress was assessed by western blot analysis by the level of p-PERK, and one of the 9 experiments for each cell type is depicted. (B) Anoxia increased the p-PERK level in all the evaluated RPTECs. Under conditions of reoxygenation, p-PERK epression remained increased in the human- and mouse-derived RPTECs, while in the hamster-derived RPTECs, it returned to the level seen under normoxic conditions. ${ }^{*} \mathrm{P}<0.05$, compared to the controls cultured under normoxic conditions; ${ }^{\wedge} \mathrm{P}<0.05$, significance between cells under reoxygenation and cells under anoxic conditions. Error bars indicate the means \pm SD. Hu, human; Mo, mouse; Ha, hamster; RPTECs, renal proximal tubular epithelial cells; p-PERK, phosphorylated protein kinase RNA-like ER kinase.

A

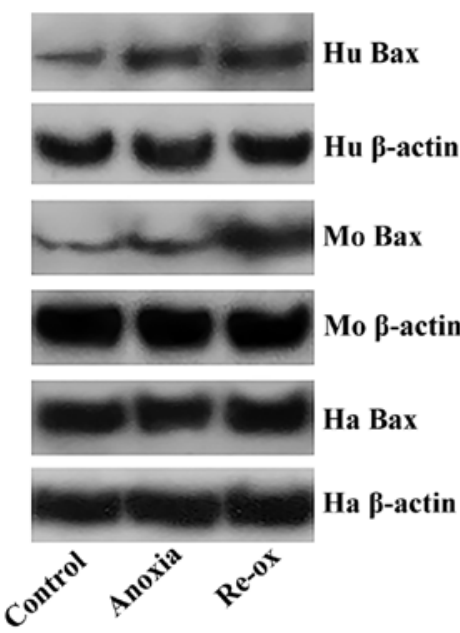

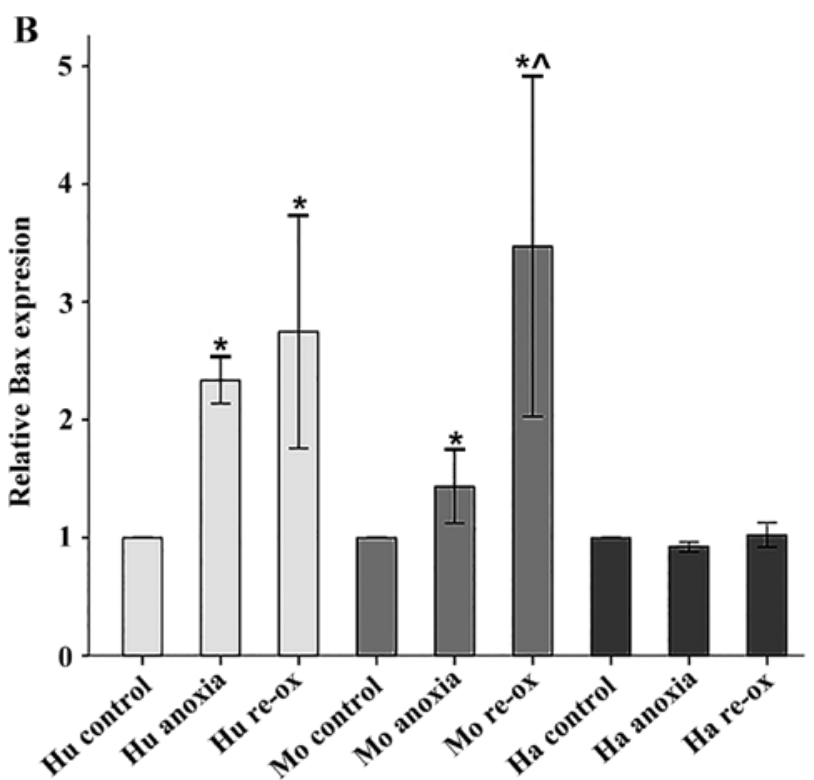

Figure 3. Bax protein expression due to anoxia or reoxygenation. Human-, mouse- or Syrian hamster-derived RPTECs were cultured under normoxic conditions or subjected to anoxia or anoxia followed by reoxygenation. (A) The expression of Bax was assessed by western blot analysis, and one of the 9 experiments for each cell type is depicted. (B) Both anoxia and reoxygenation increased Bax expression in the human- and mouse-derived RPTECs. However, neither anoxia nor reoxygenation altered the expression of Bax significantly in the hamster-derived RPTECs. " $\mathrm{P}<0.05$, compared to the controls cultured under normoxic conditions; ${ }^{\wedge} \mathrm{P}<0.05$, significance between cells under reoxygenation and cells under anoxic conditions. Error bars indicate the means $\pm \mathrm{SD}$. Hu, human; Mo, mouse; Ha, hamster; RPTECs, renal proximal tubular epithelial cells.

decreased proteasomal activity to $0.57 \pm 0.08$ of the activity seen in the control cells. On the contrary, in the hamster-derived RPTECs under conditions of reoxygenation, proteasomal activity remained significantly higher, $1.99 \pm 0.24$-fold the activity observed in the hamster cells cultured under normoxic conditions (Fig. 5).

\section{Discussion}

Comparative biology may reveal novel strategies for preventing or ameliorating human diseases. Hibernators survive repeated cycles of IR (6,7), while IR injury contributes to the pathogenesis of a number of human diseases $(1-3)$, AKI included $(4,5)$. 

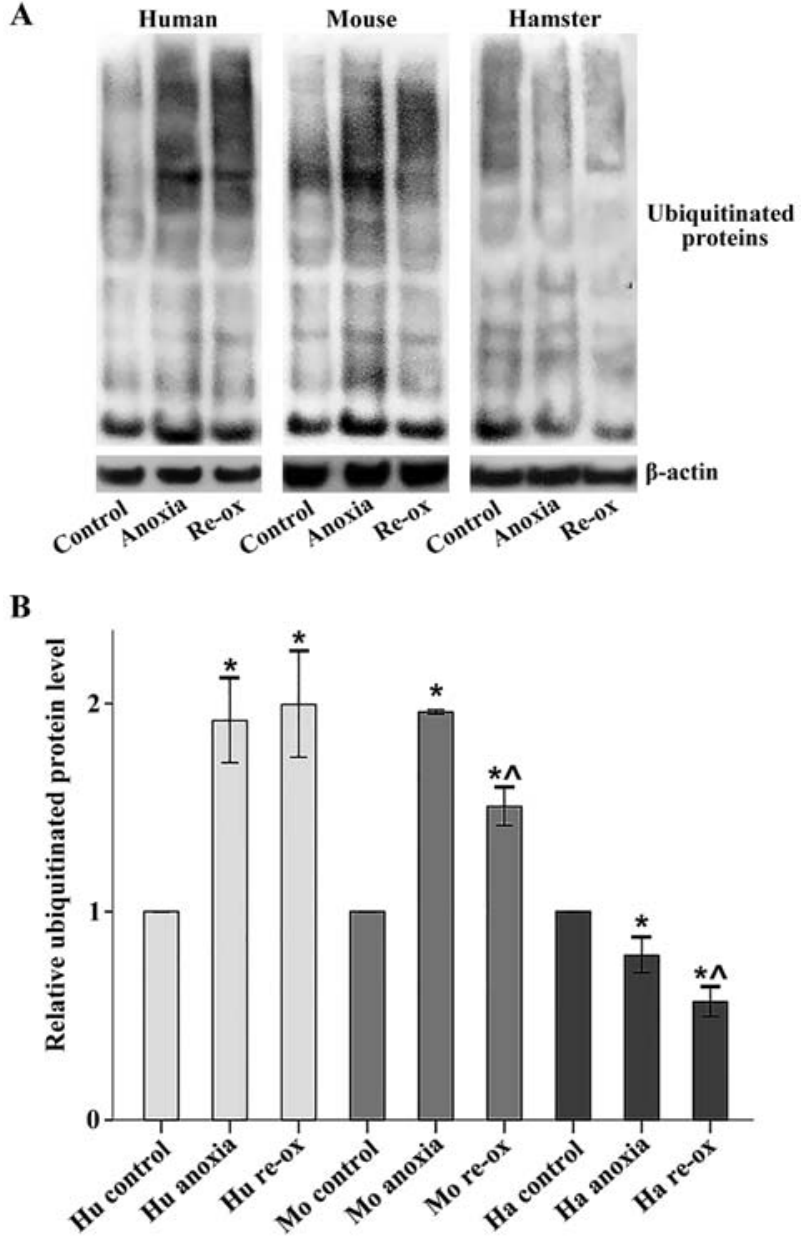

Figure 4. Ubiquitinated proteins in RPTECs under conditions of anoxia or reoxygenation. Human-, mouse- or Syrian hamster-derived RPTECs were cultured under normoxic conditions or subjected to anoxia or anoxia followed by reoxygenation. (A) Ubiquitinated proteins were assessed by western blot analysis, and one of the 9 experiments for each cell type is depicted. (B) In the human- and mouse-derived RPTECs, anoxia and reoxygenation increased the levels of ubiquitinated proteins. On the contrary, in the hamster-derived RPTECs, the levels of ubiquitinated proteins decreased slightly under anoxic conditions and considerably under conditions of reoxygenation ${ }^{*} \mathrm{P}<0.05$, compared to the controls cultured under normoxic conditions; ${ }^{\wedge} \mathrm{P}<0.05$, significance between cells under reoxygenation and cells under anoxic conditions. Error bars indicate the means \pm SD. Hu, human; Mo, mouse; Ha, hamster; RPTECs, renal proximal tubular epithelial cells.

IR induces ER stress, which may cause cell death in various cell types, including RPTECs (18-22). This study aimed to elucidate the mechanisms through which RPTECs derived from the native hibernator Syrian hamster cope with ER stress.

Compared to human or the phylogenetically related mouse cells, hamster-derived RPTECs were found to be extremely resistant to anoxia or reoxygenation-induced cell death, as assessed biochemically by LDH release assay. Similar outcomes were obtained by cell imaging experiments. These results are in accordance with those of previous studies, which have demonstrated that hibernators are less susceptible to IR injury than phylogenetically related, but unable to hibernate species $(8,9)$.

To determine whether differences in IR-induced ER stress are involved in the resistance of hamster-derived RPTECs to cell death, ER stress was assessed by evaluating the phosphorylation status of PERK. This phosphorylation is an early event

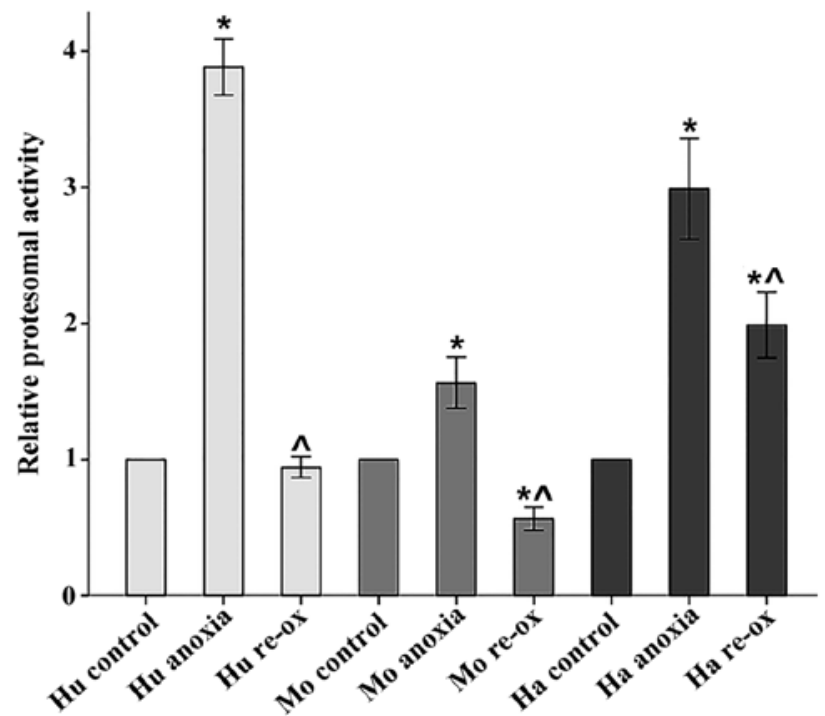

Figure 5. Proteasomal activity in RPTECs under conditions of anoxia or reoxygenation. Human-, mouse- or Syrian hamster-derived RPTECs were cultured under normoxic conditions or subjected to anoxia or anoxia followed by reoxygenation. Proteasomal activity was assessed by the proteasomal chymotrypsin-like degradation of a specific substrate. Anoxia increased proteasomal activity in all the evaluated cells. In the human-derived RPTECs, reoxygenation returned proteasomal activity to the level seen in the control cells cultured under normoxic conditions. In the mouse-derived RPTECs, reoxygenation reduced proteasomal activity below the level found in the controls. However, in the hamster-derived RPTECs, proteasomal activity remained high under conditions of eoxygenation. " $\mathrm{P}<0.05$, compared to the controls cultured under normoxic conditions; ${ }^{\wedge} \mathrm{P}<0.05$, significance between cells under reoxygenation and cells under anoxic conditions. Error bars indicate the means \pm SD. Hu, human; Mo, mouse; Ha, hamster; RPTECs, renal proximal tubular epithelial cells.

of the UPR elicited by ER stress (11). In this study, we found that anoxia induced ER stress in all the evaluated RPTECs. However, during reoxygenation, ER stress remained high in the human- and mouse-derived RPTECs, but disappeared in the hamster-derived RPTECs. Thus, the restoration of ER homeostasis in hamster-derived RPTECs during reoxygenation may contribute to their resistance to cell death. Nevertheless, there was an inconsistency between the ER stress and cell death results in the hamster-derived RPTECs under conditions of anoxia. In this case, ER stress was increased, but the hamster-derived RPTECs survived the anoxia.

Subsequently, we evaluated one of the ER stress-induced cell death pathways. Although the UPR elicited from ER stress aims to restore ER homeostasis, if the initiating stimulus is severe or prolonged, then activated PERK, IRE1 and ATF6 induce a CHOP-mediated upregulation of various pro-apoptotic proteins, including Bax $(14,15)$. Of note, while Bax is a known pro-apoptotic factor acting through outer mitochondrial membrane permeabilization, myocardial infraction experiments demonstrated that Bax also by inducing mitochondrial fusion lowers the threshold for inner membrane mitochondrial permeability transition pore opening and necrosis (23). In this study, we detected elevated levels of Bax in the human- and mouse-derived RPTECs subjected to either anoxia or reoxygenation. Conversely, and consistent with the resistance to cell death, in the hamster-derived RPTECs, neither anoxia nor reoxygenation affected Bax expression. The rapid recovery of hamster-derived RPTECs from ER stress during reoxygenation 
may explain the low Bax expression under reoxygenation. In the case of anoxia, the low Bax level in the hamster-derived RPTECs, despite the increased ER stress, could indicate a milder degree of ER stress or a more effective UPR or could be the result of other molecular mechanisms. For instance, the microRNA-21 level has been shown to increase in various tissues during hibernation $(24,25)$. Since microRNA-21 suppresses the expression of various pro-apoptotic proteins including Bax $(26,27)$, this has been suggested as a mechanism for avoiding apoptosis during hibernation (28).

Subsequently, we evaluated the ER-associated protein degradation part of the UPR. Under conditions of both anoxia and reoxygenation, the level of ubiquitinated proteins in humanand mouse-derived RPTECs increased. Conversely, the levels of ubiquitinated proteins decreased in the hamster-derived RPTECs; slightly by anoxia and considerably during reoxygenation. To explain the above results and for discriminating the role of the UPR-induced ubiquitination of misfolded proteins (12), from alterations in proteasomal activity, the latter was also evaluated. Of note, the role of proteasomal activity in IR-induced injury has not yet been elucidated completely. For instance, there is a disagreement as to whether proteasome inhibitors prevent or exacerbate IR injury-induced AKI in non-hibernating rodents $(29,30)$. In this study, anoxia increased proteasomal activity in the RPTECs from all the evaluated species. However, during reoxygenation, proteasomal activity returned to the level seen under normoxic conditions in the human-derived RPTECs, decreased below the control level in mouse-derived RPTECs, but remained high in the hamster-derived RPTECs. Notably, IR injury decreases proteasomal activity in the mouse brain and heart $(31,32)$, while during entry into torpor, the expression of certain proteasomal components increases in the liver of the hibernator golden-mantled ground squirrel (33).

As regards the human- and mouse-derived RPTECs under anoxic conditions, it is likely that the UPR-induced increase in misfolded protein ubiquitination rate overwhelms the capacity of the proteasome for their degradation despite the rise of its activity, contributing to ER stress. The interpretation of the results from hamster-derived RPTECs under anoxic conditions seems to be more complex. As in the previous cells, in the hamster-derived RPTECs, anoxia enhanced proteasomal activity, although the levels of ubiquitinated proteins slightly decreased. Whether this is the result of an adequate proteasomal function for compensating the increased protein ubiquitination, or of other mechanisms, such as a greater increase of chaperone expression in hamster than in human- or mouse-derived RPTECs in the context of the UPR (7), remains to be elucidated. As regards human- and mouse-derived RPTECs during reoxygenation, the reduction in proteasomal activity may explain the increased levels of ubiquitinated proteins and the persistent ER stress. Accordingly, the high proteasomal activity during reoxygenation in hamster-derived RPTECs may explain the decrease in the level of ubiquitinated proteins and the rapid recovery of these cells from ER stress.

In conclusion, this study demonstrates that compared to human- or mouse-derived RPTECs, RPTECs of the native hibernator hamster recover more rapidly from ER stress following warm anoxia-reoxygenation possibly, due to increased proteasomal function. This, along with other mechanisms that reduce the ER stress-induced expression of pro-apoptotic factors may contribute to the resistance of hamster-derived RPTECs to cell death due to warm anoxia-reoxygenation. Elucidating the mechanisms through which hibernators cope with IR-induced ER stress may provide clinically useful information for preventing or treating IR-induced AKI and other human diseases in the future.

\section{Acknowledgements}

Not applicable.

\section{Funding}

This study was funded only by the resources of our department.

\section{Availability of data and materials}

All data generated or analyzed during this study are included in this published article or are available from the corresponding author on reasonable request.

\section{Authors' contributions}

TE designed the study. GP and TE performed the experiments. TE, GP, GA, GF, VL and IS analyzed the results. TE wrote the manuscript with help from GP. IS provided support at all stages. All authors have read and approved the final manuscript.

\section{Ethics approval and consent to participate}

Not applicable.

\section{Patient consent for publication}

Not applicable.

\section{Competing interests}

The authors declare that they have no competing interests.

\section{References}

1. Neri M, Riezzo I,Pascale N,Pomara C and Turillazzi E: Ischemia/ reperfusion injury following acute myocardial infarction: A critical issue for clinicians and forensic pathologists. Mediators Inflamm 2017: 7018393, 2017.

2. Bakthavachalam P and Shanmugam PST: Mitochondrial dysfunction - Silent killer in cerebral ischemia. J Neurol Sci 375: 417-423, 2017.

3. Tsukamoto T, Chanthaphavong RS and Pape HC: Current theories on the pathophysiology of multiple organ failure after trauma. Injury 41: 21-26, 2010.

4. Bonventre JV and Yang L: Cellular pathophysiology of ischemic acute kidney injury. J Clin Invest 121: 4210-4221, 2011.

5. Lieberthal W and Nigam SK: Acute renal failure. I. Relative importance of proximal vs. distal tubular injury. Am J Physiol 275: F623-F631, 1998.

6. Carey HV, Andrews MT and Martin SL: Mammalian hibernation: Cellular and molecular responses to depressed metabolism and low temperature. Physiol Rev 83: 1153-1181, 2003.

7. Storey KB and Storey JM: Metabolic rate depression: The biochemistry of mammalian hibernation. Adv Clin Chem 52: $77-108,2010$ 
8. Dave KR, Prado R, Raval AP, Drew KL and Perez-Pinzon MA The arctic ground squirrel brain is resistant to injury from cardiac arrest during euthermia. Stroke 37: 1261-1265, 2006.

9. Quinones QJ, Zhang Z, Ma Q, Smith MP, Soderblom E, Moseley MA, Bain J, Newgard CB, Muehlbauer MJ, Hirschey M, et al: Proteomic profiling reveals adaptive responses to surgical myocardial ischemia-reperfusion in hibernating arctic ground squirrels compared to rats. Anesthesiology 124: 1296-1310, 2016.

10. Dausmann KH, Glos J, Ganzhorn JU and Heldmaier G: Physiology: Hibernation in a tropical primate. Nature 429 . 825-826, 2004

11. Hetz C and Papa FR: The unfolded protein response and cell fate control. Mol Cell 69: 169-181, 2018.

12. Wu X and Rapoport TA: Mechanistic insights into ER-associated protein degradation. Curr Opin Cell Biol 53: 22-28, 2018.

13. Sano R and Reed JC: ER stress-induced cell death mechanisms. Biochim Biophys Acta 1833: 3460-3470, 2013.

14. Li Y, Guo Y, Tang J, Jiang J and Chen Z: New insights into the roles of CHOP-induced apoptosis in ER stress. Acta Biochim Biophys Sin (Shanghai) 46: 629-640, 2014.

15. Oyadomari S and Mori M: Roles of CHOP/GADD153 in endoplasmic reticulum stress. Cell Death Differ 11: 381-389, 2004.

16. Maekawa $\mathrm{H}$ and Inagi R: Stress signal network between hypoxia and ER stress in chronic kidney disease. Front Physiol 8: 74, 2017.

17. Xu Y, Guo M, Jiang W, Dong H, Han Y, An XF and Zhang J: Endoplasmic reticulum stress and its effects on renal tubular cells apoptosis in ischemic acute kidney injury. Ren Fail 38: 831-837, 2016.

18. Vekich JA, Belmont PJ, Thuerauf DJ and Glembotski CC: Protein disulfide isomerase-associated 6 is an ATF6-inducible ER stress response protein that protects cardiac myocytes from ischemia/reperfusion-mediated cell death. J Mol Cell Cardiol 53: 259-267, 2012.

19. Tang JY, Jin P, He Q, Lu LH, Ma JP, Gao WL, Bai HP and Yang J: Naringenin ameliorates hypoxia/reoxygenation-induced endoplasmic reticulum stress-mediated apoptosis in $\mathrm{H} 9 \mathrm{c} 2$ myocardial cells: Involvement in ATF6, IRE1 $\alpha$ and PERK signaling activation. Mol Cell Biochem 424: 111-122, 2017.

20. Zhao Y, Fang Y, Zhao H, Li J, Duan Y, Shi W, Huang Y, Gao L and Luo Y: Chrysophanol inhibits endoplasmic reticulum stress in cerebral ischemia and reperfusion mice. Eur J Pharmacol 818: $1-9,2018$

21. Fougeray S, Bouvier N, Beaune P, Legendre C, Anglicheau D, Thervet $\mathrm{E}$ and Pallet N: Metabolic stress promotes renal tubular inflammation by triggering the unfolded protein response. Cell Death Dis 2: e143, 2011

22. Yu W, Sheng M, Xu R, Yu J, Cui K, Tong J, Shi L, Ren H and $\mathrm{Du} \mathrm{H}$ : Berberine protects human renal proximal tubular cells from hypoxia/reoxygenation injury via inhibiting endoplasmic reticulum and mitochondrial stress pathways. J Transl Med 11: $24,2013$.
23. Whelan RS, Konstantinidis K, Wei AC, Chen Y, Reyna DE, Jha S, Yang Y, Calvert JW, Lindsten T, Thompson CB, et al: Bax regulates primary necrosis through mitochondrial dynamics. Proc Natl Acad Sci USA 109: 6566-6571, 2012.

24. Morin P Jr, Dubuc A and Storey KB: Differential expression of microRNA species in organs of hibernating ground squirrels: A role in translational suppression during torpor. Biochim Biophys Acta 1779: 628-633, 2008.

25. Biggar KK and Storey KB: Identification and expression of microRNA in the brain of hibernating bats, Myotis lucifugus. Gene 544: 67-74, 2014.

26. Liu Z, Lu Y, Xiao Y and Lu Y: Upregulation of miR-21 expression is a valuable predicator of advanced clinicopathological features and poor prognosis in patients with renal cell carcinoma through the p53/p21-cyclin E2-Bax/caspase-3 signaling pathway. Oncol Rep 37: 1437-1444, 2017.

27. Gui F, Hong Z, You Z, Wu H and Zhang Y: MiR-21 inhibitor suppressed the progression of retinoblastoma via the modulation of PTEN/PI3K/AKT pathway. Cell Biol Int 40: 1294-1302, 2016.

28. Logan SM and Storey KB: Avoiding apoptosis during mammalian hibernation. Temp Austin 4: 15-17, 2016.

29. Takaoka M, Itoh M, Hayashi S, Kuro T and Matsumura Y: Proteasome participates in the pathogenesis of ischemic acute renal failure in rats. Eur J Pharmacol 384: 43-46, 1999.

30. Huber JM, Tagwerker A, Heininger D, Mayer G, Rosenkranz AR and Eller K: The proteasome inhibitor bortezomib aggravates renal ischemia-reperfusion injury. Am J Physiol Renal Physiol 297: F451-F460, 2009.

31. Keller JN, Huang FF, Zhu H, Yu J, Ho YS and Kindy TS: Oxidative stress-associated impairment of proteasome activity during ischemia-reperfusion injury. J Cereb Blood Flow Metab 20: 1467-1473, 2000.

32. Tian Z, Zheng H, Li J, Li Y, Su H and Wang X: Genetically induced moderate inhibition of the proteasome in cardiomyocytes exacerbates myocardial ischemia-reperfusion injury in mice. Circ Res 111: 532-542, 2012.

33. Epperson LE, Dahl TA and Martin SL: Quantitative analysis of liver protein expression during hibernation in the golden-mantled ground squirrel. Mol Cell Proteomics 3: 920-933, 2004.

This work is licensed under a Creative Commons Attribution-NonCommercial-NoDerivatives 4.0 International (CC BY-NC-ND 4.0) License. 